\title{
Local and global Solution of fractional Nonlinear Integro-Differential Equation
}

\author{
Ghada Shuker Jameel \\ Department of Mathematics / College of Education \\ University of Mosul
}

Received

$10 / 06 / 2013$
Accepted

$20 / 08 / 2013$

(المبخد

يتضمن البحث دراسـة وجود ووحدانية واستقرار الحل لمعادلة تكاملية-تفاضلية لاخطية

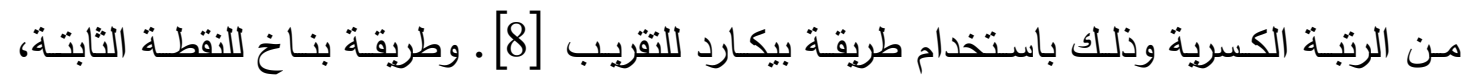

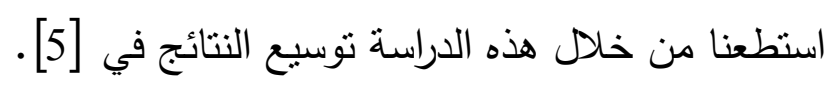

\begin{abstract}
In this paper we study the existence, uniqueness and stability solution of fractional nonlinear integro-differential equation, by using the Picard approximation method [8], and Banach fixed point method. also we extend some results obtain in [5].
\end{abstract}

\section{Introduction}

The calculation of the fractional differentiation and integration have been associated with many scientists, the most famous are Hopital L., Leibnitz G. W., Riemann B. and Liouville J.[7]. Who gave basic definitions to fractional derivative and after those, the researches continued in this area in different directions, the researcher Bassam M. A. has expanded the definition Holmgren-M. Riesz and applied the results obtained on some existence theories of ordinary differential equations [4].

The researches has rolled in fractional differential equations $[10,11]$, these include some examples such as:

Both the researchers Al-Abedeen A. Z. and Arora H. L. [1] studied existence and uniqueness solution for fractional differential equations as following:

$x^{\alpha}(t)=f(t, x) \quad, \quad x^{\alpha-1}\left(t_{0}\right)=x_{0} \quad, \quad 0<\alpha \leq 1$

Using Banach's way for fixed point. 
The researchers Butris, R. N. and Hussen Abdul-Qader, M. A. [5] studied Some results in theory integro-differential equation of fractional order, as following:

$$
x^{\alpha}(t)=f\left(t, x, \int_{-\infty}^{t} G(t, s) g(s, x(s)) d s\right) \quad, \quad x^{\alpha-1}(0)=x_{0} \quad, \quad 0<\alpha<1
$$

And the researchers Moulay Rchid Sidi Ammi, El Hassan El Kinani, Delfim F. M. Torres [9], studied Existence and uniqueness of solution to a functional integro-differential fractional equation, as following:

$$
\frac{d^{\alpha}}{d t^{\alpha}}\left[\frac{x(t)}{f(t, x(t))}\right]=g\left(t, x_{t}, \int_{0}^{t} k\left(s, x_{s}\right) d s\right) \quad \text { a.e., } \quad t \in I \quad, \quad x(t)=\Phi(t), \quad t \in I_{0}
$$

But our work is to study existence, uniqueness and stability solution of fractional nonlinear integro-differential equation as following:

$$
\begin{aligned}
& x^{\alpha}(t)=A(t) x(t)+\int_{0}^{t} K(t, s) F(t, s, x(s, x)) d s+f(t) \\
& x_{0}^{\alpha-1}\left(t, x_{0}\right)=x_{0} \quad, \quad 0<\alpha \leq 1
\end{aligned}
$$

In this paper we set some definitions and lemmas to be used in the proof of the main theorem.

\section{Definition 1 [6]:}

Let $\mathrm{f}$ be a function which is defined a. e. (almost every where) on $[a, b]$. For $\alpha>0$, we define:

$$
\mathrm{I}_{a}^{b} f=\frac{1}{\Gamma(\alpha)} \int_{a}^{b}(b-s)^{\alpha-1} f(s) d s
$$

Provided that this integral (Lebesgue) exists.

Definition 2 [6]:

If $\alpha>0$, then Gamma's function is denote by $(\Gamma)$ and defined by the form: $\Gamma(\alpha)=\int_{0}^{\infty} e^{-s} s^{\alpha-1} d s$

\section{Lemma 1 [2]:}

If $\left\{f_{n}\right\}_{n=1}^{\infty}$ is a sequences of functions is defined on the set $E \subseteq R$ such that $\left|f_{n}\right| \leq M_{n}$, where $M_{n}$ is a positive number, then $\sum_{n=1}^{\infty} f_{n}$ is uniformaly convergent on $E$ if $\sum_{n=1}^{\infty} M_{n}$ is convergent. 


\section{Lemma 2 [3]:}

Let $E_{\alpha}(m ; x)=\sum_{m=1}^{\infty} \frac{m^{n-1} x^{n \alpha-1}}{\Gamma(n \alpha)}$, where $\mathrm{m}=\mathrm{R}$, then:

1. the series converges for $x \neq 0$ and $\alpha>0$.

2. the series converges everywhere when $\alpha \geq 0$.

3 . if $\alpha=0$, then $E_{1}(m, x)=\exp (m x)$.

\section{Lemma 3:}

If $K_{1}$ and $K_{2}$ be a positive constant, and $f$ be a continuous function on $a \leq t \leq b$, such that:

Then

$$
f(t) \leq K_{1}+K_{2} \int_{a}^{t} f(s) d s
$$

$$
f(t) \leq K_{1} \exp \left(K_{2}(t-a)\right)
$$

From Picard approximation method we can studying the solution of fractional nonlinear integro-differential equation, as the form:

$$
\begin{aligned}
& x^{\alpha}(t)=A(t) x(t)+\int_{0}^{t} K(t, s) F(t, s, x(s, x)) d s+f(t) \\
& x_{0}^{\alpha-1}\left(t, x_{0}\right)=x_{0} \quad, \quad 0<\alpha \leq 1
\end{aligned}
$$

where the function $F(t, s, x(t, x))$ is a continuous in $t$, and satisfies Lipschitz. Condition in $x$ and defined on the domain: $(t, s, x) \in[0, T] \times[0, T] \times G_{\alpha}$

Where $x \in G_{\alpha} \subseteq[0, T]$ and $G_{1 \alpha}$ is a closed and bounded domain.

Suppose that the function $F(t, s, x(t, x)), f(t)$ satisfies the following inequalities:

$$
\begin{aligned}
& \|F(t, s, x)\| \leq M \quad, \quad\|f(t)\| \leq N \\
& \left\|F\left(t, s, x_{1}\right)-F\left(t, s, x_{2}\right)\right\| \leq L\left\|x_{1}-x_{2}\right\|,
\end{aligned}
$$

for all $t \in[0, T], s \in[0, T]$ and $x, x_{1}, x_{2} \in G_{\alpha}$, where $L, M, N$, are positive constants.

Let $A(t), K(t, s)$ are positive matrices $n \times n$, defined in (1.2), and continuous at $t, s$ and satisfies the following inequalities:

$$
\begin{aligned}
& \|K(t, s)\| \leq H \\
& \left\|e^{\int_{0}^{t} A(\eta) d \eta}\right\| \leq Q
\end{aligned}
$$

Where $0 \leq s \leq t \leq T$ and $H, Q$ are positive constants. 
We define the non-empty sets as follows:

$G_{\alpha f}=G_{\alpha}-\frac{T^{\alpha}}{\Gamma(\alpha+1)} M_{1}$

where $M_{1}=Q[H M T+N], \Lambda=Q H L T$ and $\|\|=.\max |$.$| .$

\section{Existence Solution}

The study of the existence solution of the problem (1.1) will be introduced by the following:

\section{Theorem 1:}

Let the function $F(t, s, x(t, x))$ be defined in the domain (1.2), continuous in $t, x$ and satisfy the inequalities (1.3), (1.4), (1.5) and (1.6), then the function:

$$
x\left(t, x_{0}\right)=\frac{x_{0} e^{0} t(\eta) d \eta}{\Gamma(\alpha)}+\frac{1}{\Gamma(\alpha)} \int_{0}^{t} e^{t} e^{0} A(\eta) d \eta\left[\int_{0}^{s} K(s, \tau) F\left(s, \tau, x\left(\tau, x_{0}\right)\right) d \tau+f(s)\right](t-s)^{\alpha-1} d s
$$

Is solution for the equation (1.1).

\section{Proof:}

Let

$x_{m+1}\left(t, x_{0}\right)=\frac{x_{0} e^{0} A(\eta) d \eta}{\Gamma(\alpha)} t^{\alpha-1}+\frac{1}{\Gamma(\alpha)} \int_{0}^{t} e^{t} \int^{t} A(\eta) d \eta\left[\int_{0}^{s} K(s, \tau) F\left(s, \tau, x_{m}\left(\tau, x_{0}\right)\right) d \tau+f(s)\right](t-s)^{\alpha-1} d s$

with

$x_{0}^{\alpha-1}\left(t, x_{0}\right)=x_{0} e^{\int^{t} A(\eta) d \eta} \quad, \quad m=0,1,2, \ldots$

be a sequence of functions defined on the domain:

$\left(t, x_{0}\right) \in[0, T] \times G_{\alpha f}$

we will divided the proof as follows:

(i) $x_{m}\left(t, x_{0}\right) \in G_{\alpha}$, for all $t \in[0, T], x_{0} \in G_{\alpha f}$.

(ii) $x_{m}\left(t, x_{0}\right) \in G_{\alpha}$, is uniformly convergent to the function $x\left(t, x_{0}\right)$ on (2.3), for all $t \in[0, T], x_{0} \in G_{\alpha f}$.

(iii) $x\left(t, x_{0}\right) \in G_{\alpha}$, for all $t \in[0, T], x_{0} \in G_{\alpha f}$.

\section{proof (i):}

Set $\mathrm{m}=0$ and use (2.2), we get: 


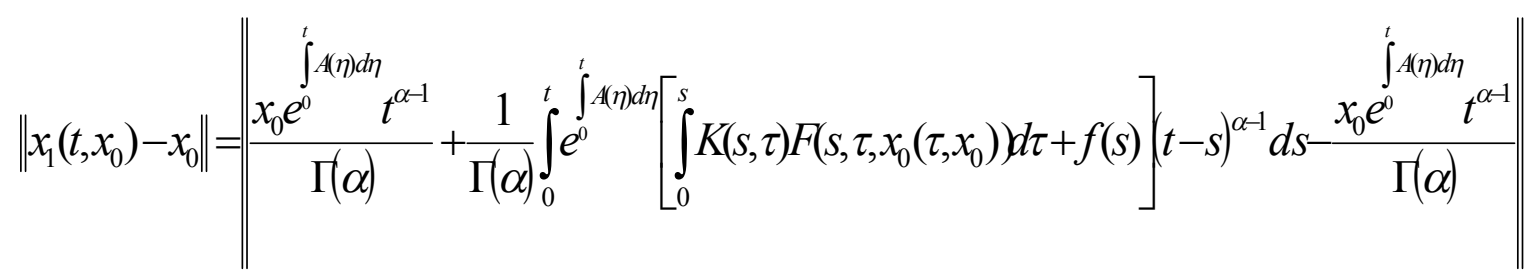

$$
\begin{aligned}
& \leq \frac{1}{\Gamma(\alpha)} \int_{0}^{t}\left\|\int^{t} A(\eta) d \eta\right\|\left[\int^{s}\|K(s, \tau)\| F\left(s, \tau, x_{0}\left(\tau, x_{0}\right)\right)\|d \tau+\| f(s) \|(t-s)^{\alpha-1} d s\right. \\
& \leq \frac{1}{\Gamma(\alpha)} \int_{0}^{t} Q\left[\int_{0}^{s} H M d \tau+N\right](t-s)^{\alpha-1} d s \\
& \leq \frac{1}{\Gamma(\alpha)} \int_{0}^{t} Q[H M T+N](t-s)^{\alpha-1} d s \\
& \leq \frac{1}{\Gamma(\alpha)} \int_{0}^{t} M_{1}(t-s)^{\alpha-1} d s \\
& \leq \frac{t^{\alpha}}{\Gamma(\alpha+1)} M_{1} \quad, \quad t \in[0, T] \\
& \left\|x_{1}\left(t, x_{0}\right)-x_{0}\right\| \leq \frac{T^{\alpha}}{\Gamma(\alpha+1)} M_{1}
\end{aligned}
$$

That is $x_{1}\left(t, x_{0}\right) \in G_{\alpha}$, for all $t \in[0, T], x_{0} \in G_{\alpha f}$.

By induction we have:

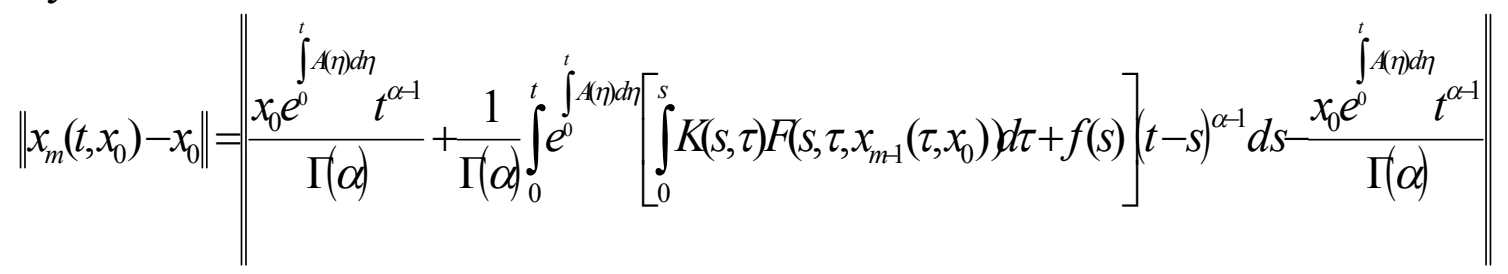

$\left\|x_{m}\left(t, x_{0}\right)-x_{0}\right\| \leq \frac{T^{\alpha}}{\Gamma(\alpha+1)} M_{1}$

where $x_{m}\left(t, x_{0}\right) \in G_{\alpha}$, for all $t \in[0, T], x_{0} \in G_{\alpha f}$.

proof (ii):

We prove now that the sequence (2.2) is uniformly convergent in (2.3). From (2.2), when $\mathrm{m}=1$ we get:

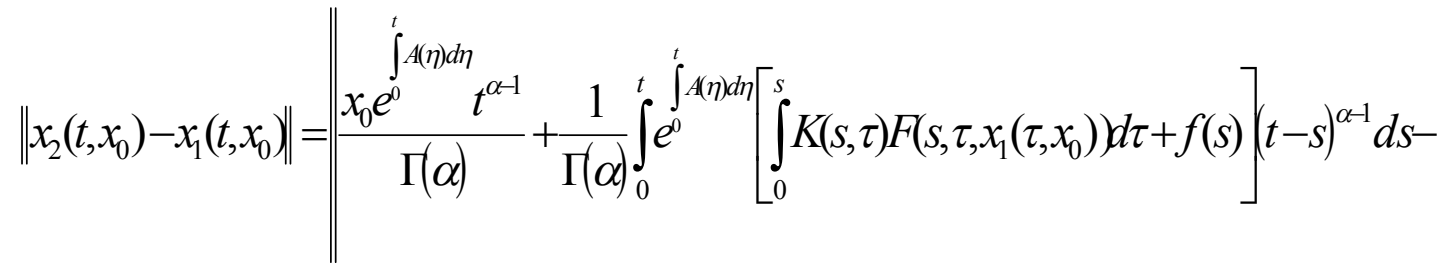

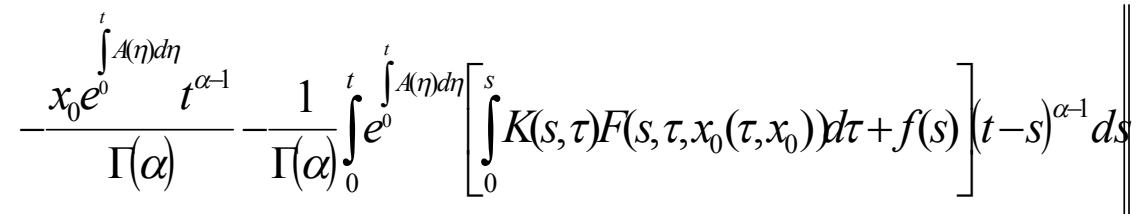




$$
\begin{aligned}
& \leq \frac{1}{\Gamma(\alpha)} \int_{0}^{t} Q\left[\int_{0}^{s} H L\left\|x_{1}\left(\tau, x_{0}\right)-x_{0}\left(\tau, x_{0}\right)\right\| d \tau\right](t-s)^{\alpha-1} d s \\
& \leq \frac{1}{\Gamma(\alpha)} \int_{0}^{t} Q\left[\int_{0}^{s} H L \frac{M_{1}}{\Gamma(\alpha+1)} T^{\alpha} d \tau\right](t-s)^{\alpha-1} d s \\
& \leq \frac{1}{\Gamma(\alpha)} \frac{M_{1}}{\Gamma(\alpha+1)} T^{\alpha} \int_{0}^{t} Q H L T(t-s)^{\alpha-1} d s \\
& =\frac{M_{1}}{\Gamma(\alpha+1)} T^{\alpha} \Lambda \frac{1}{\Gamma(\alpha)} \int_{0}^{t}(t-s)^{\alpha-1} d s \\
& \leq\left(\frac{T^{\alpha}}{\Gamma(\alpha+1)}\right)^{2} M_{1} \Lambda
\end{aligned}
$$

therefore

$$
\left\|x_{2}\left(t, x_{0}\right)-x_{1}\left(t, x_{0}\right)\right\| \leq\left(\frac{T^{\alpha}}{\Gamma(\alpha+1)}\right)^{2} M_{1} \Lambda
$$

Now when $\mathrm{m}=2$ in (2.2) we get the following:

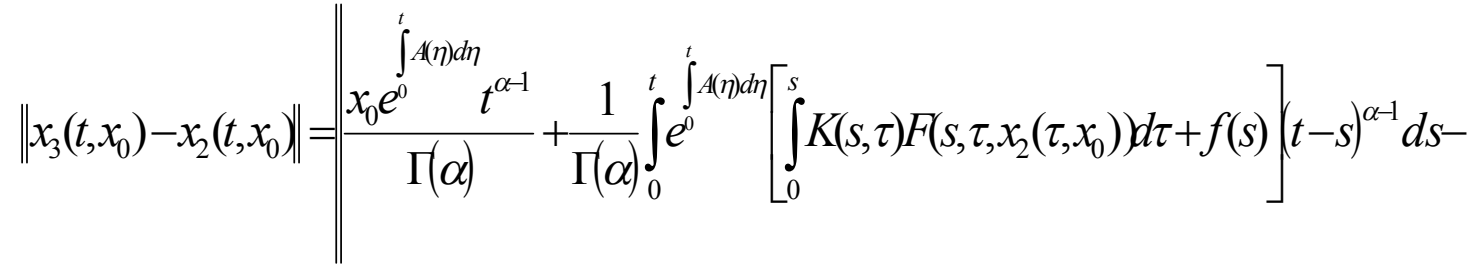

$$
\begin{aligned}
& -\frac{x_{0} e^{e^{t}} t^{\alpha-1}}{\Gamma(\alpha)} \frac{1}{\Gamma(\alpha)} \int_{0}^{t} e^{t} e^{0}(\eta \eta) d \eta\left[\int_{0}^{s} K(s, \tau) F\left(s, \tau, x_{1}\left(\tau, x_{0}\right)\right) d \tau+f(s)\right](t-s)^{\alpha-1} d s \\
& \leq \frac{1}{\Gamma(\alpha)} \int_{0}^{t} Q\left[\int_{0}^{s} H L\left(\frac{T^{\alpha}}{\Gamma(\alpha+1)}\right)^{2} M_{1} \Lambda d \tau\right](t-s)^{\alpha-1} d s \\
& \leq\left(\frac{T^{\alpha}}{\Gamma(\alpha+1)}\right)^{2} \frac{1}{\Gamma(\alpha)} M_{1} \Lambda \int_{0}^{t} Q H L T(t-s)^{\alpha-1} d s \\
& =\left(\frac{T^{\alpha}}{\Gamma(\alpha+1)}\right)^{2} M_{1} \Lambda^{2} \frac{1}{\Gamma(\alpha)} \int_{0}^{t}(t-s)^{\alpha-1} d s \\
& \leq\left(\frac{T^{\alpha}}{\Gamma(\alpha+1)}\right)^{3} M_{1} \Lambda^{2}
\end{aligned}
$$


therefore

$$
\left\|x_{3}\left(t, x_{0}\right)-x_{2}\left(t, x_{0}\right)\right\| \leq\left(\frac{T^{\alpha}}{\Gamma(\alpha+1)}\right)^{3} M_{1} \Lambda^{2}
$$

By induction we have:

$\left\|x_{m+1}\left(t, x_{0}\right)-x_{m}\left(t, x_{0}\right)\right\| \leq\left(\frac{T^{\alpha}}{\Gamma(\alpha+1)}\right)^{m+1} M_{1} \Lambda^{m}$

for $\mathrm{m}=0,1,2, \ldots$.

Now from (2.7), and for $P \geq 1$, we get:

$\left\|x_{m+p}\left(t, x_{0}\right)-x_{m}\left(t, x_{0}\right)\right\| \leq M_{1} \sum_{i=0}^{p-1}\left(\frac{T^{\alpha}}{\Gamma(\alpha+1)}\right)^{i+1} \Lambda^{i}$

where

$$
\begin{array}{r}
\left\|x_{m+p}\left(t, x_{0}\right)-x_{m}\left(t, x_{0}\right)\right\|=\left\|x_{m+p}\left(t, x_{0}\right)-x_{m+p-1}\left(t, x_{0}\right)\right\|+ \\
+\left\|x_{m+p-1}\left(t, x_{0}\right)-x_{m+p-2}\left(t, x_{0}\right)\right\|+\ldots \ldots \ldots \ldots+ \\
+\left\|x_{m+1}\left(t, x_{0}\right)-x_{m}\left(t, x_{0}\right)\right\| \\
\leq\left(\frac{T^{\alpha}}{\Gamma(\alpha+1)} \Lambda\right)^{m+p-1}\left\|x_{1}\left(t, x_{0}\right)-x_{0}\right\| \\
+\left(\frac{T^{\alpha}}{\Gamma(\alpha+1)} \Lambda\right)^{m+p-2}\left\|x_{1}\left(t, x_{0}\right)-x_{0}\right\|+ \\
+\ldots \ldots+\left(\frac{T^{\alpha}}{\Gamma(\alpha+1)} \Lambda\right)^{m}\left\|x_{1}\left(t, x_{0}\right)-x_{0}\right\|
\end{array}
$$

where

$$
\begin{array}{r}
\left\|x_{m+p}\left(t, x_{0}\right)-x_{m}\left(t, x_{0}\right)\right\| \leq\left(\frac{T^{\alpha}}{\Gamma(\alpha+1)} \Lambda\right)^{m}\left[1+\left(\frac{T^{\alpha}}{\Gamma(\alpha+1)} \Lambda\right)+\left(\frac{T^{\alpha}}{\Gamma(\alpha+1)} \Lambda\right)^{2}+\ldots .\right. \\
+\left(\frac{T^{\alpha}}{\Gamma(\alpha+1)} \Lambda\right)^{p-2}+\left(\frac{T^{\alpha}}{\Gamma(\alpha+1)} \Lambda\right)^{p-1}\left\|x_{1}\left(t, x_{0}\right)-x_{0}\right\|
\end{array}
$$

We note that the right hand from (2.9) is bounded with convergent geometric series it's summation is $\frac{1}{1-\Lambda}$ we get:

$$
\begin{array}{r}
\left\|x_{m+p}\left(t, x_{0}\right)-x_{m}\left(t, x_{0}\right)\right\| \leq\left(\frac{T^{\alpha}}{\Gamma(\alpha+1)} \Lambda\right)^{m}\left[1-\left(\frac{T^{\alpha}}{\Gamma(\alpha+1)} \Lambda\right)\right]^{-1}\left\|x_{1}\left(t, x_{0}\right)-x_{0}\right\| \\
\leq\left(\frac{T^{\alpha}}{\Gamma(\alpha+1)} \Lambda\right)^{m}\left[1-\left(\frac{T^{\alpha}}{\Gamma(\alpha+1)} \Lambda\right)\right]^{-1} \frac{T^{\alpha}}{\Gamma(\alpha+1)} M_{1} \Lambda
\end{array}
$$


for $\left(\frac{T^{\alpha}}{\Gamma(\alpha+1)} \Lambda\right)<1, P \geq 1$

then

$$
\operatorname{Lim}_{m \rightarrow \infty}\left(\frac{T^{\alpha}}{\Gamma(\alpha+1)} \Lambda\right)^{m}=0
$$

So that the rights hand from (2.10) equal zero when $m \rightarrow \infty$. Suppose that $\varepsilon>O$, we get a positive integer $n$ such that $n<m$, and satisfied the next estimation for all $m$ : $\left\|x_{m+p}\left(t, x_{0}\right)-x_{m}\left(t, x_{0}\right)\right\|<\varepsilon, \quad$ for $P \in N$.

Then according to the definition of uniformly convergent, we find that the sequence $\left\{x_{m}\left(t, x_{0}\right)\right\}_{m=0}^{\infty}$ is uniformly convergent from the function $x\left(t, x_{0}\right)$ and this function be continuous on the same interval. We put:

$$
\operatorname{Lim}_{m \rightarrow \infty} x_{m}\left(t, x_{0}\right)=x_{\infty}\left(t, x_{0}\right)
$$

\section{proof (iii):}

to prove $x\left(t, x_{0}\right) \in G_{\alpha}$, for all $t \in[0, T], x_{0} \in G_{\alpha f}$ we take:

$$
\begin{gathered}
\| \frac{1}{\Gamma(\alpha)} \int_{0}^{t} e^{t} A(\eta) d \eta\left[\int_{0}^{s} K(s, \tau) F\left(s, \tau, x_{m}\left(\tau, x_{0}\right)\right) d \tau+f(s)\right](t-s)^{\alpha-1} d s- \\
\quad-\frac{1}{\Gamma(\alpha)} \int_{0}^{t} \int^{t} A(\eta) d \eta\left[\int_{0}^{s} K(s, \tau) F\left(s, \tau, x\left(\tau, x_{0}\right)\right) d \tau+f(s)\right](t-s)^{\alpha-1} d s \| \\
\leq \frac{\Lambda}{\Gamma(\alpha)} \int_{0}^{t}\left\|x_{m}\left(s, x_{0}\right)-x\left(s, x_{0}\right)\right\|(t-s)^{\alpha-1} d s
\end{gathered}
$$

Now

$$
\begin{aligned}
\lim _{m \rightarrow \infty} \| \frac{1}{\Gamma(\alpha)} \int_{0}^{t} e^{\int_{0}} A(\eta) d \eta & {\left[\int_{0}^{s} K(s, \tau) F\left(s, \tau, x_{m}\left(\tau, x_{0}\right)\right) d \tau+f(s)\right](t-s)^{\alpha-1} d s-} \\
& -\frac{1}{\Gamma(\alpha)} \int_{0}^{t} \int^{t} A(\eta) d \eta\left[\int_{0}^{s} K(s, \tau) F\left(s, \tau, x\left(\tau, x_{0}\right)\right) d \tau+f(s)\right](t-s)^{\alpha-1} d s \mid \\
& \leq \lim _{m \rightarrow \infty} \frac{\Lambda}{\Gamma(\alpha)} \int_{0}^{t}\left\|x_{m}\left(s, x_{0}\right)-x\left(s, x_{0}\right)\right\|(t-s)^{\alpha-1} d s
\end{aligned}
$$


Since the sequence $\left\{x_{m}\left(t, x_{0}\right)\right\}_{m=0}^{\infty}$ is uniformly convergent on $[0, T]$ from the function $x\left(t, x_{0}\right)$ on the same interval.

$$
\begin{aligned}
\lim _{m \rightarrow \infty} \frac{1}{\Gamma(\alpha)} \int_{0}^{t} e^{t} A(\eta) d \eta\left[\int_{0}^{s} K(s, \tau) F\left(s, \tau, x_{m}\left(\tau, x_{0}\right)\right) d \tau+f(s)\right](t-s)^{\alpha-1} d s= \\
=\frac{1}{\Gamma(\alpha)} \int_{0}^{t} e^{t} A(\eta) d \eta\left[\int_{0}^{s} K(s, \tau) F\left(s, \tau, x\left(\tau, x_{0}\right)\right) d \tau+f(s)\right](t-s)^{\alpha-1} d s
\end{aligned}
$$

So $x\left(t, x_{0}\right) \in G_{\alpha}$, for all $x_{0} \in G_{\alpha f}$

\section{Uniqueness solution}

The study of the uniqueness solution of the problem (1.1), will be introduced by the following:

\section{Theorem 2:}

Let all assumptions and conditions of theorem 1 be given then the problem (1.1), has a unique solution $x=x_{\infty}\left(t, x_{0}\right)$ on the domain (2.3).

\section{Proof:}

We have to show to that $x\left(t, x_{0}\right)$ is a unique solution of problem (1.1). On the contrary, we suppose that there is two different solutions $x\left(t, x_{0}\right)$ and $\hat{x}\left(t, x_{0}\right)$ of the problem (1.1), defined in the form:

$$
\hat{x}\left(t, x_{0}\right)=\frac{x_{0} e^{\int^{t} A(\eta) d \eta} t^{\alpha-1}}{\Gamma(\alpha)}+\frac{1}{\Gamma(\alpha)} \int_{0}^{t} e^{t} A(\eta) d \eta\left[\int_{0}^{s} K(s, \tau) F\left(s, \tau, \hat{x}\left(\tau, x_{0}\right)\right) d \tau+f(s)\right](t-s)^{\alpha-1} d s
$$

Now we will prove that $\hat{x}\left(t, x_{0}\right)=x\left(t, x_{0}\right)$ for $x_{0} \in G_{\alpha f}$, by prove the following inequality:

$$
\left\|\hat{x}\left(t, x_{0}\right)-x_{m}\left(t, x_{0}\right)\right\| \leq\left(\frac{T^{\alpha}}{\Gamma(\alpha+1)}\right)^{m+1} M_{1}^{*} \Lambda^{m}
$$

$$
\text { where } M_{1}^{*}=Q[H R T+N], R=\max _{t \in[0, T]}\|F(s, t, \hat{x})\| \text {. }
$$

Let when $m=0$ in (2.2) and from (3.1) we find:

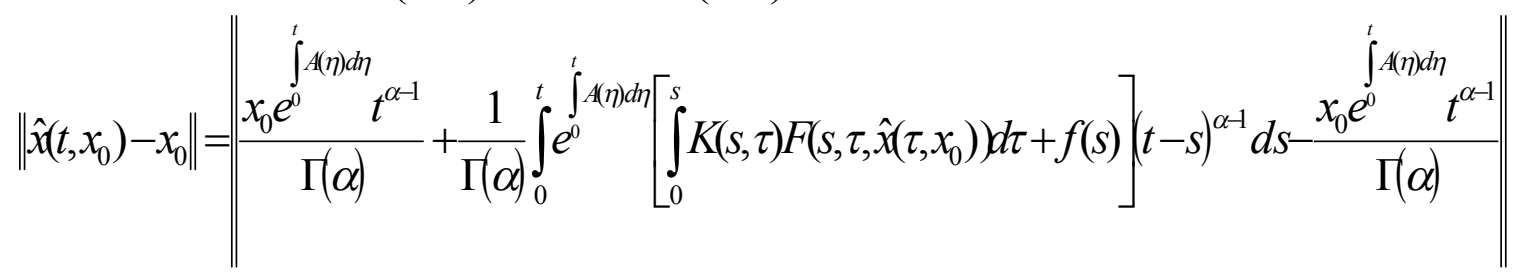

$$
\begin{aligned}
& \leq \frac{1}{\Gamma(\alpha)} \int_{0}^{t}\left\|\int^{\int^{0} A(\eta) d t}\right\|\left[\int_{0}^{s}\|K(s, \tau)\|\left\|F\left(s, \tau, \hat{x}\left(\tau, x_{0}\right)\right)\right\| d \tau+\|f(s)\|(t-s)^{\alpha-1} d s\right.
\end{aligned}
$$




$$
\begin{aligned}
& \leq \frac{1}{\Gamma(\alpha)} \int_{0}^{t} Q\left[\int_{0}^{s} H R d \tau+N\right](t-s)^{\alpha-1} d s \\
& \leq \frac{1}{\Gamma(\alpha)} \int_{0}^{t} Q[H R T+N](t-s)^{\alpha-1} d s \\
& \leq \frac{1}{\Gamma(\alpha)} \int_{0}^{t} M_{1}^{*}(t-s)^{\alpha-1} d s \\
& \leq \frac{t^{\alpha}}{\Gamma(\alpha+1)} M_{1}^{*}, \quad t \in[0, T] \\
\left\|x_{1}\left(t, x_{0}\right)-x_{0}\right\| & \leq \frac{T^{\alpha}}{\Gamma(\alpha+1)} M_{1}^{*}
\end{aligned}
$$

and when $\mathrm{m}=1$ in (2.2) and from (3.1) we find:

$$
\begin{aligned}
& \left\|\hat{x}\left(t, x_{0}\right)-x_{1}\left(t, x_{0}\right)\right\|=\| \frac{x_{0} e^{0} t(\eta) d \eta}{\Gamma(\alpha)} t^{\alpha-1}+\frac{1}{\Gamma(\alpha)} \int_{0}^{t} e^{t} A(\eta) d \eta\left[\left[\int_{0}^{s} K(s, \tau) F\left(s, \tau, \hat{x}\left(\tau, x_{0}\right)\right) d \tau+f(s)\right](t-s)^{\alpha-1} d s^{-}\right. \\
& \left.-\frac{x_{0} e^{0} t^{t} A(\eta) d \eta}{\Gamma(\alpha)}+\frac{1}{\Gamma(\alpha)} \int_{0}^{t} \int^{t} e^{0} A(\eta) d \eta\left[\int_{0}^{s} K(s, \tau) F\left(s, \tau, x_{0}\left(\tau, x_{0}\right)\right) d \tau+f(s)\right] t-s\right)^{\alpha-1} d s \\
& \leq \frac{1}{\Gamma(\alpha)} \int_{0}^{t} Q\left[\int_{0}^{s} H L\left\|\hat{x}\left(\tau, x_{0}\right)-x_{0}\left(\tau, x_{0}\right)\right\| d \tau\right](t-s)^{\alpha-1} d s \\
& \leq \frac{1}{\Gamma(\alpha)} \frac{M_{1}^{*}}{\Gamma(\alpha+1)} T^{\alpha} \int_{0}^{t} Q H L T(t-s)^{\alpha-1} d s \\
& \leq\left(\frac{T^{\alpha}}{\Gamma(\alpha+1)}\right)^{2} M_{1}^{*} \Lambda
\end{aligned}
$$

therefore

$$
\left\|\hat{x}\left(t, x_{0}\right)-x_{1}\left(t, x_{0}\right)\right\| \leq\left(\frac{T^{\alpha}}{\Gamma(\alpha+1)}\right)^{2} M_{1}^{*} \Lambda
$$

we find that the inequality (3.2) is satisfying when $\mathrm{m}=0,1,2$.

Suppose that the inequality (3.2) is satisfying when $\mathrm{m}=\mathrm{p}$ as the following inequality: 
$\left\|\hat{x}\left(t, x_{0}\right)-x_{p}\left(t, x_{0}\right)\right\| \leq\left(\frac{T^{\alpha}}{\Gamma(\alpha+1)}\right)^{p+1} M_{1}^{*} \Lambda^{p}$

Next we will proof the following inequality:

$\left\|\hat{x}\left(t, x_{0}\right)-x_{p+1}(t, x)\right\| \leq\left(\frac{T^{\alpha}}{\Gamma(\alpha+1)} \Lambda\right)^{p+1}\left[1-\frac{T^{\alpha}}{\Gamma(\alpha+1)} \Lambda\right]^{-1} \frac{T^{\alpha}}{\Gamma(\alpha+1)} M_{1}^{*}$

now

$$
\begin{aligned}
& \left\|\hat{x}\left(t, x_{0}\right)-x_{p+1}\left(t, x_{0}\right)\right\|=\| \frac{x_{0} e^{0} t(\eta) d \eta}{\Gamma(\alpha)}+\frac{1}{\Gamma(\alpha)} \int_{0}^{t} e^{0} \int^{t} A(\eta) d \eta\left[\int_{0}^{s} K(s, \tau) F\left(s, \tau, \hat{x}\left(\tau, x_{0}\right)\right) d \tau+f(s)\right](t-s)^{\alpha-1} d s- \\
& -\frac{x_{0} e^{\int^{0} A(\eta) d \eta} t^{\alpha-1}}{\Gamma(\alpha)}+\frac{1}{\Gamma(\alpha)} \int_{0}^{t} \int^{t} A(\eta) d \eta\left[\int_{0}^{s} K(s, \tau) F\left(s, \tau, x_{p}\left(\tau, x_{0}\right)\right) d \tau+f(s)\right](t-s)^{\alpha-1} d s \\
& \leq \frac{1}{\Gamma(\alpha)} \int_{0}^{t} Q\left[\int_{0}^{s} H L\left\|\hat{x}\left(\tau, x_{0}\right)-x_{p}\left(\tau, x_{0}\right)\right\| d \tau\right](t-s)^{\alpha-1} d s \\
& \leq \frac{1}{\Gamma(\alpha)} \int_{0}^{t} Q\left[\int_{0}^{s} H L\left(\frac{T^{\alpha}}{\Gamma(\alpha+1)}\right)^{p+1} M_{1}^{*} \Lambda^{p} d \tau\right](t-s)^{\alpha-1} d s \\
& \leq\left(\frac{T^{\alpha}}{\Gamma(\alpha+1)}\right)^{p+1} M_{1}^{*} \Lambda^{p+1} \frac{1}{\Gamma(\alpha)} \int_{0}^{t}(t-s)^{\alpha-1} d s \\
& \leq\left(\frac{T^{\alpha}}{\Gamma(\alpha+1)}\right)^{p+2} M_{1}^{*} \Lambda^{p+1}
\end{aligned}
$$

then

$$
\left\|\hat{x}\left(t, x_{0}\right)-x_{p+1}\left(t, x_{0}\right)\right\| \leq\left(\frac{T^{\alpha}}{\Gamma(\alpha+1)}\right)^{p+2} M_{1}^{*} \Lambda^{p+1}
$$

Thus we find that the inequality (3.2) is satisfying when $\mathrm{m}=0,1,2, \ldots$.

Then by a condition (2.12) we get:

$\hat{x}\left(t, x_{0}\right)=\operatorname{Lim}_{m \rightarrow \infty} x_{m}\left(t, x_{0}\right)=x\left(t, x_{0}\right)$

and this prove that the two solutions are congruent in the domain (2.3).

\section{Stability solution}

The study of the stability solution of the problem (1.1), will be introduced by the following theorem: 


\section{Theorem 3:}

If the inequalities (1.3), (1.4), (1.5) and (1.6), were satisfied, and $z\left(t, x_{0}\right)$, which was defined bellow as different solutions for the equation (1.1), then the solution was stabile if satisfy the inequality:

$$
\left|x_{0}\left(0, x_{0}\right)-z\left(0, x_{0}\right)\right|<\delta \quad, \quad \delta<0
$$

Where

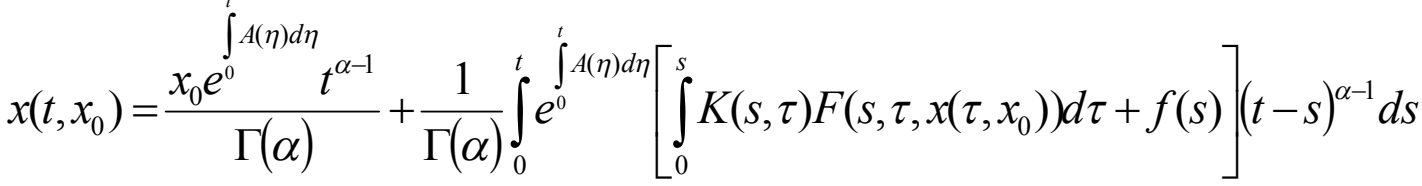

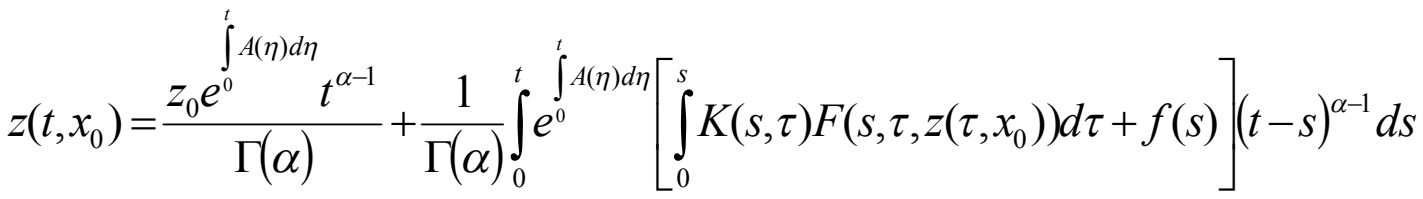

proof:

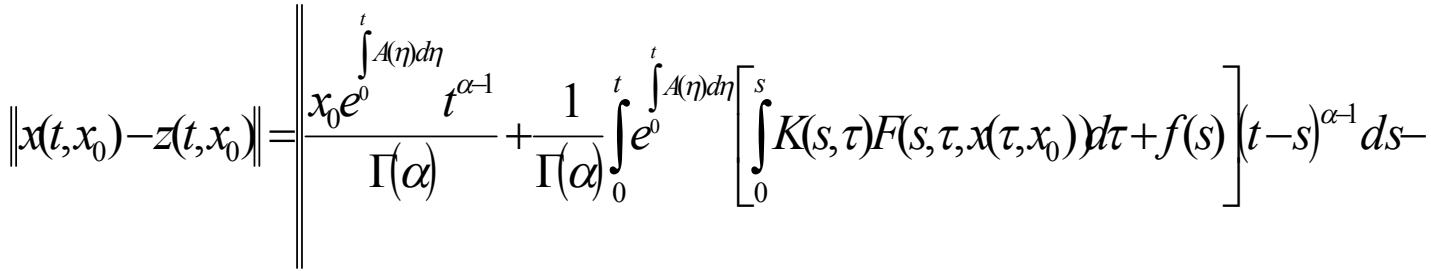

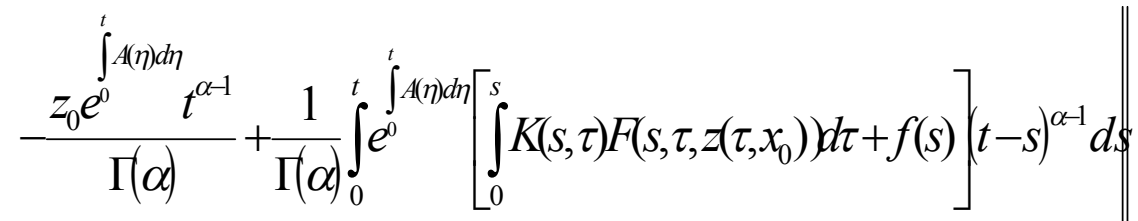

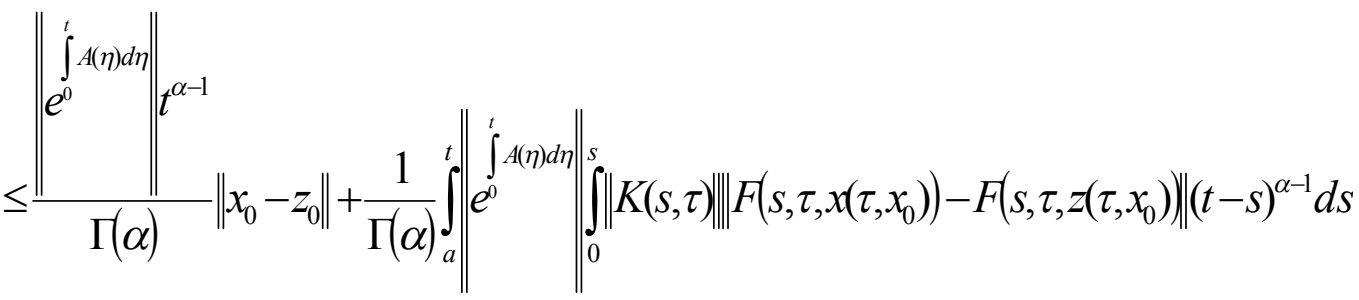

$$
\begin{aligned}
& \leq \frac{Q t^{\alpha-1}}{\Gamma(\alpha)}\left\|x_{0}-z_{0}\right\|+\frac{1}{\Gamma(\alpha)} \int_{0}^{t} Q\left[\int_{0}^{s} H L\left\|x\left(\tau, x_{0}\right)-z\left(\tau, x_{0}\right)\right\| d \tau\right](t-s)^{\alpha-1} d s
\end{aligned}
$$

Let $\left\|x_{0}-z_{0}\right\| \leq \delta$, we get:

$$
\begin{aligned}
& \leq \frac{Q t^{\alpha-1}}{\Gamma(\alpha)} \delta+\frac{1}{\Gamma(\alpha)} \int_{0}^{t} Q H L T\left\|x\left(s, x_{0}\right)-z\left(s, x_{0}\right)\right\|(t-s)^{\alpha-1} d s \\
& \leq \frac{Q t^{\alpha-1}}{\Gamma(\alpha)} \delta+\frac{1}{\Gamma(\alpha)} \Lambda \int_{0}^{t}\left\|x\left(s, x_{0}\right)-z\left(s, x_{0}\right)\right\|(t-s)^{\alpha-1} d s
\end{aligned}
$$

Let $\frac{Q t^{\alpha-1}}{\Gamma(\alpha)} \delta=\delta_{1} \quad, \quad$ and by the lemma 3, we get: 
$\left\|x\left(t, x_{0}\right)-z\left(t, x_{0}\right)\right\| \leq \delta_{1} \exp \left[\frac{t^{\alpha} \Lambda}{\Gamma(\alpha+1)}\right]$

Put $\quad \exp \left[\frac{t^{\alpha} \Lambda}{\Gamma(\alpha+1)}\right]=\frac{\varepsilon}{\delta_{1}}$

$\left\|x\left(t, x_{0}\right)-z\left(t, x_{0}\right)\right\| \leq \delta_{1} \frac{\varepsilon}{\delta_{1}}$

$\left\|x\left(t, x_{0}\right)-z\left(t, x_{0}\right)\right\| \leq \varepsilon$

then the solution was stabile in the given domain.

\section{Banach method}

The investigation of Banach method of the problem (1.1), will be introduced by the following theorem:

\section{Theorem 4:}

Let $(s,\|\cdot\|)$, define a mapping $\mathrm{T}^{*}$ on $G_{\alpha}$ as:

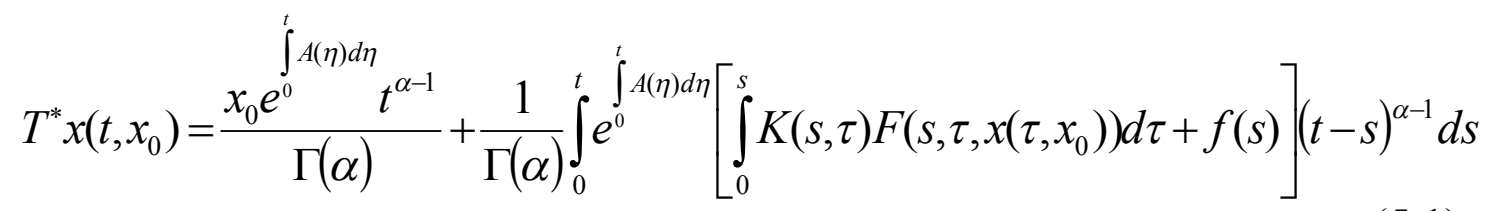

Since the equation (5.1) defined in the domain (1.2), continuous in $t, x$ and satisfy the inequalities:

$$
\begin{aligned}
& |F(t, s, x)| \leq M \quad, \quad|f(t)| \leq N \\
& \left|F\left(t, s, x_{1}\right)-F\left(t, s, x_{2}\right)\right| \leq L\left|x_{1}-x_{2}\right|, \\
& |K(t, s)| \leq H \\
& \left|\int^{0} A(\eta) d \eta\right| \leq Q
\end{aligned}
$$

then $T^{*} \in G_{\alpha}$, and hence $T^{*}: G_{\alpha} \rightarrow G_{\alpha}$, Next we claim that $\mathrm{T}^{*}$ is contraction mapping.

\section{Proof:}

Let $x, z \in G_{\alpha}$, then

$$
\begin{aligned}
& \left\|T^{*} x\left(t, x_{0}\right)-T^{*} z\left(t, x_{0}\right)\right\|=\mid \frac{x_{0} e^{0} t(\eta) d \eta}{\Gamma(\alpha)}+\frac{1}{\Gamma(\alpha)} \int_{0}^{t} e^{\int^{0} A(\eta) d \eta}\left[\int_{0}^{s} K(s, \tau) F\left(s, \tau, x\left(\tau, x_{0}\right)\right) d \tau+f(s)\right](t-s)^{\alpha-1} d s^{-} \\
& -\frac{z_{0} e^{0} t^{\alpha-1} A(\eta) d \eta}{\Gamma(\alpha)}+\frac{1}{\Gamma(\alpha)} \int_{0}^{t} e^{\int_{0}^{0} A(\eta) d \eta}\left[\int_{0}^{s} K(s, \tau) F\left(s, \tau, z\left(\tau, x_{0}\right)\right) d \tau+f(s)\right](t-s)^{\alpha-1} d s
\end{aligned}
$$




$$
\begin{aligned}
& \leq \frac{\left|e^{\int^{0} A(\eta) d \eta}\right| t^{\alpha-1}}{\Gamma(\alpha)}\left|x_{0}-z_{0}\right|+\frac{1}{\Gamma(\alpha)} \int_{a}^{t}\left|e^{0} e^{0} A(\eta) d \eta\right|\left|\int_{0}^{s}\right| K(s, \tau)\left|F\left(s, x\left(s, x_{0}\right)\right)-F\left(s, z\left(s, x_{0}\right)\right)\right|(t-s)^{\alpha-1} d s \\
& \leq \frac{Q t^{\alpha-1}}{\Gamma(\alpha)}\left|x_{0}-z_{0}\right|+\frac{1}{\Gamma(\alpha)} \int_{0}^{t} Q\left[\int_{0}^{s} H L\left|x\left(\tau, x_{0}\right)-z\left(\tau, x_{0}\right)\right| d \tau\right](t-s)^{\alpha-1} d s \\
& \leq \frac{Q t^{\alpha-1}}{\Gamma(\alpha)}\left|x_{0}-z_{0}\right|+\frac{1}{\Gamma(\alpha)} \int_{0}^{t} Q H L t\left|x\left(s, x_{0}\right)-z\left(s, x_{0}\right)\right|(t-s)^{\alpha-1} d s \\
& \leq \frac{Q t^{\alpha-1}}{\Gamma(\alpha)}\left|x_{0}-z_{0}\right|+\frac{t^{\alpha}}{\Gamma(\alpha+1)} \Lambda\left|x\left(t, x_{0}\right)-z\left(t, x_{0}\right)\right|
\end{aligned}
$$

let

$$
\frac{Q t^{\alpha-1}}{\Gamma(\alpha)}\left|x_{0}-z_{0}\right| \leq \sigma\left|x\left(t, x_{0}\right)-z\left(t, x_{0}\right)\right|
$$

we get:

$$
\begin{aligned}
& \left\|T^{*} x\left(t, x_{0}\right)-T^{*} z\left(t, x_{0}\right)\right\| \leq \sigma\left|x\left(t, x_{0}\right)-z\left(t, x_{0}\right)\right|+\frac{t^{\alpha}}{\Gamma(\alpha+1)} \Lambda\left|x\left(t, x_{0}\right)-z\left(t, x_{0}\right)\right| \\
& \text { so }\left\|T^{*} x\left(t, x_{0}\right)-T^{*} z\left(t, x_{0}\right)\right\|=\max _{t}\left|T^{*} x\left(t, x_{0}\right)-T^{*} z\left(t, x_{0}\right)\right|
\end{aligned}
$$

there fore:

$$
\begin{aligned}
\max _{t}\left|T^{*} x\left(t, x_{0}\right)-T^{*} z\left(t, x_{0}\right)\right| & \leq \max _{t}\left[\sigma\left|x\left(t, x_{0}\right)-z\left(t, x_{0}\right)\right|+\frac{t^{\alpha}}{\Gamma(\alpha+1)} \Lambda\left|x\left(t, x_{0}\right)-z\left(t, x_{0}\right)\right|\right] \\
& =\max _{t}\left(\sigma+\frac{t^{\alpha}}{\Gamma(\alpha+1)} \Lambda\right)\left|x\left(t, x_{0}\right)-z\left(t, x_{0}\right)\right|
\end{aligned}
$$

Suppose that $\psi=\left(\sigma+\frac{t^{\alpha}}{\Gamma(\alpha+1)} \Lambda\right)$, where $0<\psi<1$, then

$$
\max _{t}\left|T^{*} x\left(t, x_{0}\right)-T^{*} z\left(t, x_{0}\right)\right|=\psi \max _{t}\left|x\left(t, x_{0}\right)-z\left(t, x_{0}\right)\right|
$$

So $\left\|T^{*} x\left(t, x_{0}\right)-T^{*} z\left(t, x_{0}\right)\right\| \leq \psi\left\|x\left(t, x_{0}\right)-z\left(t, x_{0}\right)\right\|$

and hence $\mathrm{T}^{*}$ is contraction mapping, and $\mathrm{T}^{*}$ has a fixed point $x \in S$. i.e

$T^{*} x\left(t, x_{0}\right)=\frac{x_{0} e^{\int^{t} A(\eta) d \eta} t^{\alpha-1}}{\Gamma(\alpha)}+\frac{1}{\Gamma(\alpha)} \int_{0}^{t} e^{t} A(\eta) d \eta\left[\int_{0}^{s} K(s, \tau) F\left(s, \tau, x\left(\tau, x_{0}\right)\right) d \tau+f(s)\right](t-s)^{\alpha-1} d s$ $x\left(t, x_{0}\right)$ is a unique solution of (1.1). 


\section{Remark:}

In this paper Banach method is Local solution and Picard method is global solution for the equation (1.1).

\section{REFERENCES}

1) Al-abedean A. Z., Arora H. L., "A global existence and uniqueness theorem for ordinary differential equation at generalized order", Canada, J. Math. No. 21, (1978).

2) Apostal, Tom M., "Mathematical Analysis", $2^{\text {nd }}$ edition AddisionWesley publishing Company, Inc, London, (1964).

3) Barrett, J. H. "Differential equation of non-integer order" Canada J. Math. Vol. 6, (1954), P.529-541.

4) Bassam, M. A., "Some existence theorem on differential equation of generalized order", Canada, J. Math. 10, (1965).

5) Butris, R. N. and Hussen Abdul-Qader, M. A. "Some results in theory integro-differential equation of fractional order", Iraq, Mosul, J. of Educ. And sci, Vol.49, (2001), p.88-98.

6) Hadid S. B. "Local and global existence theorems on differential equations of non integer order", J. of Fractional Calculus Vol. 7, (1995).

7) Keith, B. Oldham and Spanier "The fractional calculus" N. Y., (1974).

8) Struble, R. A., "Nonlinear differential equations", McGraw-Hill Book Company, Inc, New York, (1962).

9) Moulay Rchid Sidi Ammi, El Hassan El Kinani, Delfim F. M. Torres, "Existence and uniqueness of solution to a functional integro-differential fractional equation" Electronic Journal of Differential Equations, (2012).

10) Xinguang Zhang, Lishan Liu, Yonghong $\mathrm{Wu}$, Yinan Lu "The iterative solutions of nonlinear fractional differential equations" Applied Mathematics and Computation 219 (2013) 4680-4691.

11) Xinguang Zhang, Yuefeng Han "Existence and uniqueness of positive solutions for higher order nonlocal fractional differential equations" Applied Mathematics Letters 25 (2012) 555-560. 